\title{
RE-ENGINEERING OF SMART CITY'S BUSINESS PROCESSES BASED ON SOCIAL NETWORKS AND INTERNET OF THINGS
}

\author{
$U D C((004.738+304.5): 383.12)$
}

\author{
Emir Ugljanin', Dragan Stojanović ${ }^{2}$, \\ Ejub Kajan ${ }^{1}$, Zakaria Maamar ${ }^{3}$ \\ ${ }^{1}$ State University of Novi Pazar, Republic of Serbia \\ ${ }^{2}$ University of Niš, Niš, Republic of Serbia \\ ${ }^{3}$ Zayed University, Dubai, United Arab Emirates
}

\begin{abstract}
This paper reports our experience with developing a Business-2-Social (B2S) platform that provides necessary support to all this platform's constituents, namely business processes, social media (e.g., social network), and Internet of Things (IoT). This platform is exemplified with smart cities whose successful management requires a complete integration of IoT and social media capabilities into the business processes implementing user services. To ensure a successful integration, social actions, that a smart city would allow citizens execute, are analyzed in terms of impact of these smart city's business processes. Reactions to these actions are tracked and then analyzed to improve user services.
\end{abstract}

Key words: business process, Internet of Things, smart city, social network.

\section{INTRODUCTION}

In Europe, 70 percent of the population already lives in urban areas and 80 percent will be living there by 2020 [1]. The concept of a Smart City (SC) is becoming a reality and many governments around the world are working towards making this reality happen. Examples of European cities with smart city strategies include Amsterdam, Barcelona, London, and Stockholm [2]. Along with government bodies, some industrial partners like Cisco have been engaged in building smart cities like Songdo in South Korea that is " $a$ prime example of a new city that brings together the world's best technologies, building design and eco-friendly practices to create the ultimate lifestyle and work experience" ${ }^{\text {. }}$ Despite the abundance of literature on smart cities, there is no formal definition of what a smart city is, could be, or should be. Some buzzwords used are "cyberville", "information city", "intelligent city", "electronic community", "ubiquitous city”, "digital city”, etc.

Received October 20, 2017

Corresponding author: Emir Ugljanin

State University of Novi Pazar, Republic of Serbia

E-mail: emirugljanin@gmail.com

${ }^{1}$ newsroom.cisco.com/songdo. 
An SC relies strongly on Information and Communication Technologies (ICT) to improve the quality of life of citizens by offering cost-effective and eco-friendly services anytime, anywhere. The abundance and affordability of Internet of Things (IoT) devices such as sensors and actuators support this offering. According to Gartner ${ }^{2}$, billions of connected things were in use in 2016, up 30\% from 2015, and will reach 20.8 billion by 2020. Although IoT devices are used as primary data sources for SC, there are other sources of data that engineers of IoT-based services could tap into, for instance, social media. According to the Vice President of Intel's Architecture Group Kirk Skaugen, there was more data transmitted over the Internet in 2010 than in the entire history of the Internet up to $2009^{3}$. Bauman et al. [3] argue the "... In particular, any individual with a smart phone and a social media account can become a human "social sensor", capable of a wide range of functions, including producing data about spontaneous events in realtime". The success of SC services is dependent on how citizens' needs and requirements are met. To this end, capturing their concerns, collecting their feedback, and gauging their satisfactions are of paramount importance. Social media exemplified with social networks like Facebook ${ }^{4}$ and Twitter ${ }^{5}$ could sustain this success. Facebook, as one of the social network sites, had about 1.79 billion monthly active users in September 2016, an increase of 16 percent year-over-year [4]. Not so long ago, in 2009 Facebook had 175 million of active users [5], which implies that the number of Facebook's active users has increased more than 10 times in 7 years. Having in mind that every single user is a potential data generator, we can imagine the amount of data generated every second.

Considering the amount of raw data (i.e., unprocessed) available via IoT as well as on social networks, SC are better positioned to transform citizens' lives. Marsa-Maestre et al. [6] are expecting that SC would respond to citizens' changing concerns by adjusting and customizing their services. To this end, re-engineering the business processes ( $a k a$ know-how) that underpin these services is a must.

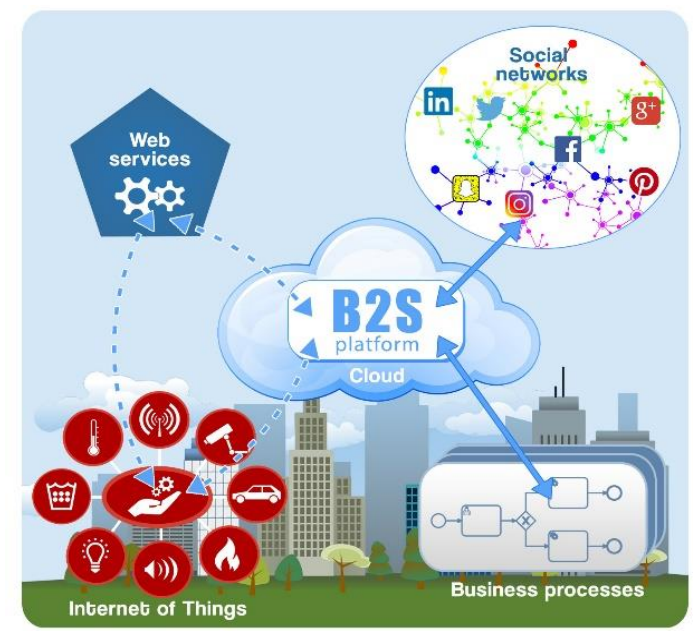

Fig. 1 Smart city's constituents

\footnotetext{
${ }^{2}$ www.gartner.com/newsroom/id/3165317.

${ }^{3}$ mashable.com/2011/10/20/kirk-skaugen-web-2.

${ }^{4}$ www.facebook.com.

${ }^{5}$ www.twitter.com
} 
Collecting, processing, and analyzing collected data provides necessary information and then, knowledge for SC's business processes that will act upon all things sensed in certain surroundings (e.g., adjusting traffic lights and diming lights). A case study of 31 informational world cities [7] has shown that governments are very active on social networks (mainly on Facebook and Twitter). It has also shown that governments need to "provoke" comments and discussion about their cities, and this is what we are working on.

In this paper, we report our experience in developing a Business-2-Social (B2S) platform that provides a necessary support to all the constituents of this platform, namely business processes, social media, and IoT. Although many available software systems and platforms leverage social networks to collect social data, we propose a different approach that automates the collection and processing of citizens' feedback in preparation for taking the necessary measures such as re-engineering the concerned business processes. We only target feedback on social actions that are executed over social media by a reserved set of citizens who subscribe to certain SC's services.

This research aims at integrating IoT sensing capabilities as a trigger for certain automatic activities like publishing campaigns on social networks and assessing the appropriateness and usefulness of these actions. Decision makers would be in a better position to improve SC's services. Figure 1 illustrates our platform for supporting SC capture citizens' concerns and act on these concerns.

This paper's contributions are manifold: (i) design of an architecture for weaving IoT into SC's social media and business processes, (ii) use of IoT data (sensors used in a wider sense, i.e., physical, virtual, social sensors - Internet/Web of Things) as initiators for starting social activities, and (iii) refinement and adaptation of business processes based on feedback collected on these activities.

The rest of this paper is structured as follows. Section 2 is an overview of the previous work on SC, IoT and social media. Section 3 presents a motivating example. Section 4 discusses some actions that social applications allow people to execute. Sections 5 present the B2S platform in terms of architecture and operations. Section 6 is about the implementation of the B2S platform. Section 7 concludes the paper and lists some future work.

\section{RELATED WORK}

Cities have deployed different IoT systems and IT infrastructures to support their SC initiatives. San Francisco (SF Park) uses smart sensors to update users with timely information so that waiting time for parking space is minimized [8]. Environmental data such as air quality, temperature, and rainfall are collected by Supertrees in Singapore ${ }^{6}$. In another example, outdoor street lighting points are configured in the city of Barcelona, with eco-digital LED technology and multi-purpose sensors, to be used as a network by multiple vendors [9]. Almost 17 years ago, the city of Southampton in the UK announced to be the first smart city after developing its multi-application smartcard for transportation, recreation, and leisure-related transactions [1]. Cities like Barcelona, Amsterdam, Berlin, Manchester, Edinburgh, and Bath are supported by the European Union to encourage more SC initiatives that would tackle energy shortages, traffic congestion, infrastructure, health, and education [10].

\footnotetext{
${ }^{6}$ www.gardensbythebay.com.sg/en/attractions/supertree-grove/facts-and-figures.html.
} 
Atlantic Re:think in partnership with Microsoft explored how cloud affects transformation of smart cities around the world ${ }^{7}$. Different areas of implementation in cities around the world are considered, such as transportation, health care, education, water and public safety. There are many frameworks and initiatives that work on provisioning IoT data from SC. Some of the examples are ODAA (Open Data Aarhuss) and iCity. ODAA ${ }^{8}$ platform provides open data access to data collected from the City of Aarhus using IoT infrastructure deployed within the city [11]. iCity ${ }^{9}$ which provides a platform for accessing data generated from services and multiple heterogeneous sensors installed in different locations in several European cities. It allows accessing all infrastructures through REST API.

The Smart Santander ${ }^{10}$ project envisions deployment of 20.000 sensors in Belgrade, Guildforf, Lübeck and, Santander, exploiting large variety of technologies. For instance, in Belgrade they use public transportation vehicles to monitor a set of environmental parameters over a large area, as well as to provide additional information about transportation to its users [12].

Other initiatives use social media for improving city services. Chaniotakis et al. [13] analyze data from social media, Facebook, Foursquare, and Twitter to be used in transportation. The Livehoods project [14] is redrawing neighborhood boundaries based on similarities in user mobility using data from Foursquare.

Some initiatives combine both IoT and social media. Badii et al. [15] worked on CityScripts that combines social networks and IoT in a SC. Although CityScripts uses social networks in combination with data from different sensors and actuators, social network usage is restricted to posting only. Another example of SC frameworks which uses near real-time IoT data and social media, is CityPulse [11]. Although CityPulse enables cross-domain data integration, their approach of analyzing social media streams from Twitter focusses on analyzing content using machine learning. They are considering all streams on some subject which can be misleading.

Despite the multiple initiatives on SC, automating social data collection in preparation for making decisions by re-engineering business processes, remains unstudied, to the best of our knowledge.

\section{MOTIVATING EXAMPLE}

Being the focus of the variety of business, fashion, sport, science, and cultural activities, cities have difficulties in dealing with the large number of visitors who "flock" to them and then, put pressure on their infrastructures, services, facilities, etc. Successful management of visitors' activities like where and when to go, what to do, how to respond, etc., should help provide better experiences to all. Using social networks and IoT, SC can do a better job!

We consider a Smart City Tourism Organization (SCTO). It embraces social media with emphasis on social networks, Facebook for instance, and has several followers on its official Facebook page ${ }^{11}$ mainly used for promotion. Until now, SCTO's Facebook page has been updated manually based on interactions with citizens and visitors. SCTO would

\footnotetext{
${ }^{7}$ www.theatlantic.com/sponsored/microsoft-2017/city-in-the-cloud/1133/

${ }^{8}$ www.odaa.dk

${ }^{9}$ www.icityproject.eu.

${ }^{10}$ www.smartsantander.eu.

${ }^{11}$ www.facebook.com/SmartCityTouristicOrganization/
} 
like to automate its business processes related to visitor mobility by communicating with them using social networks and collecting their feedback.

To avoid reducing SC performance dealing with huge number of visitors and improve quality of their visits in Smart City, SCTO needs to "communicate" with Smart City services, gather valuable information and help in changing city's daily routines. SCTO needs to assure that visitors will have enough parking space at the desired time and find the best route to avoid traffic jams. Although this is a valuable contribution, we will not analyze it, as it is currently out of scope of this paper.

Although process automation is the ultimate goal, some tasks and parameters still need user intervention at start-up time. SCTO/designated-managers rely on guidelines consisting of:

- External data, which will be used as a trigger for publishing campaigns on social media. This data should be gathered from IoT, online services, environment conditions and events, such as weather conditions, air pollution, season of the year, city events, city landmarks, occasions, followers' interest, etc.

- Social actions copy (copy is product of copywriting ${ }^{12}$ ), containing promotional materials like "hook" and "selling/offering" campaigns. Hook aims at driving subscribers' attention and checking their interest. The Hook campaigns will be published on Facebook prior to publishing selling/offering campaigns.

- Scenarios, which would allow deciding on when to post campaigns, who should respond to online feedback, what content should be posted, etc. These scenarios will refer to specific conditions linked to external data, e.g., if temperature is above 30 degrees and there are at least 50 responses to a hook campaign in 24 hours then publish selling campaign.

\section{SOCIAL ACTIONS ON SOCIAL NETWORKS}

We use the term social action in this paper, which is defined in [16] as an operation that a Web 2.0 application allows users to execute. Social actions that are available on Facebook $^{13}$, Twitter ${ }^{14}$ and Instagram ${ }^{15}$ are listed in Table 1.

There is a number of popular social networks that offer different functionalities and target different audiences, from teenagers to elderly people. Despite their popularity, each network has a set of limitations such as maximum number of characters in Twitter, only video content for Snapchat ${ }^{16}$, only images for Instagram, and only certain actions for Facebook. Despite these limitations, a set of similarities can be drawn with respect to the social actions that they allow users to execute. We have identified the following categories of actions:

- Initiation, the first action to which the other social actions could be applied.

- Response, a reaction to an initial social action which could provide some additional meta data or trigger back-and-forth interactions.

- Promotion, forwarding the outcomes of initial social actions' execution that someone has triggered and making it a new initial social action.

\footnotetext{
${ }^{12}$ en.wikipedia.org/wiki/Copywriting

${ }^{13}$ www.facebook.com

${ }^{14}$ www.twitter.com

${ }^{15}$ www.instagram.com

${ }^{16}$ www.snapchat.com.
} 
Table 1 illustrates the correspondence of some actions to certain well-known social networks.

Table 1 Social actions of social networks

\begin{tabular}{llll}
\hline $\begin{array}{l}\text { Categories } \\
\text { of actions }\end{array}$ & Facebook & Twitter & Instagram \\
\hline $\begin{array}{l}\text { Initiation } \\
\text { Response }\end{array}$ & $\begin{array}{l}\text { Post } \\
\text { comment, reply, like, reactions } \\
\text { (love, HaHa, WoW, angry, sad) }\end{array}$ & $\begin{array}{l}\text { Tweet } \\
\text { reply, like }\end{array}$ & post \\
Promotion & share, send a message & retweet, quote tweet & send to \\
\hline
\end{tabular}

To the best of our knowledge, there is no metric that would help measure the impact of executing such social actions on businesses and the other way around, i.e., how could businesses influence the choice of social actions to execute. In this paper, social action executors of our interest include SC and citizens. SC will be in charge only for initiation category of social actions, and collecting feedback from citizens. Citizens respond with social actions that fall into category of response and promotion.

\section{B2S PLATFORM'S CONCEPTS AND OPERATIONS}

The proposed B2S platform revolves around 4 axes: social world, IoT world, business process world (know-how), and their interaction.

The social world refers to specific social networks that are of interest to those in charge of managing SC. The IoT world represents sensors, actuators, and other relevant devices that contribute to developing an ecosystem of things. The know-how world represents the business processes that define the services of SCs. Finally, the B2S platform acts as a glue between them and provide their interaction.

\subsection{B2S architecture}

The B2S architecture consists of five components and four repositories (Fig.2). Below are the details of each component and repository.

- Social actions copy ${ }^{17}$ repository stores the necessary content that system uses for initiating social actions on social networks. In the context of SCTO, examples of such social actions include promoting traditional festival or events.

- Scenarios repository stores guidelines which are checked prior to publishing a social action.

- Social action trigger executes social actions on a social network.

- Social action monitor use means for parsing information about social actions executed on social networks prior to storing them in the social responses repository.

- Social responses repository stores data about all social actions which are executed on a specific social network account/page. Examples of social responses include commenting some post or sharing it.

- IoT Connector uses means for accessing data from IoT world and stores it in IoT Data repository.

${ }^{17}$ en.wikipedia.org/wiki/Copy_(written). 
- IoT Data repository stores collected data from the IoT world. Example of such data includes movement sensor values or weather data.

- B2S Analyzer is a central module responsible for making decisions whether new social action will be published or not, based on IoT data and results of previously published social actions.

- B2S Manager allows managing available repositories by administrative users, namely scenarios, social actions copy, IoT data, and social responses.

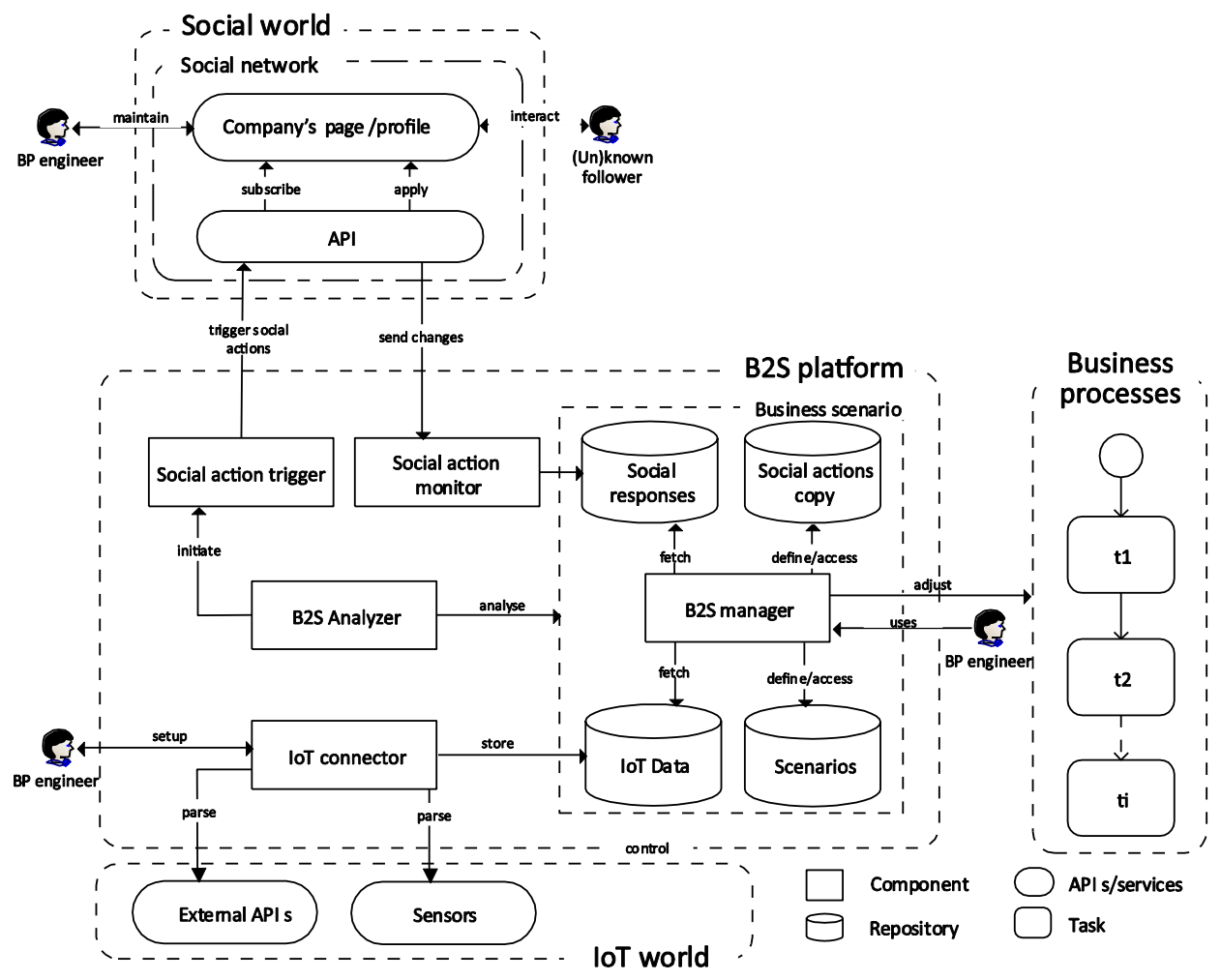

Fig. 2 General architecture of the B2S platform

Fig. 3 presents dynamics of B2S which are described below.

- Automated and regular check of preconditions and postconditions, as well as collecting (gathering) data from external sources is scheduled.

- Data gathered from cities' air pollution sensors, as well as weather data from external web services is compared to defined preconditions for choosing the right "hook" campaign from social actions copy repository based on defined scenario.

- After publishing a hook campaign, Facebook page subscribers' reactions are tracked and analyzed for predefined time, and then compared to predefined expected results (postconditions) from the scenarios repository.

- When predefined postcondition thresholds are fulfilled, automated package tour campaign will be published on the Facebook page, which is linked to the content 
of interest on the Facebook page. This could also be a trigger for changing smart city business processes.

- As far as expected results are concerned, if followers do not show interest in the published promotional campaign in some defined period of time, the system will publish a different promotional text for other city landmarks and tours.

\subsection{B2S in action}

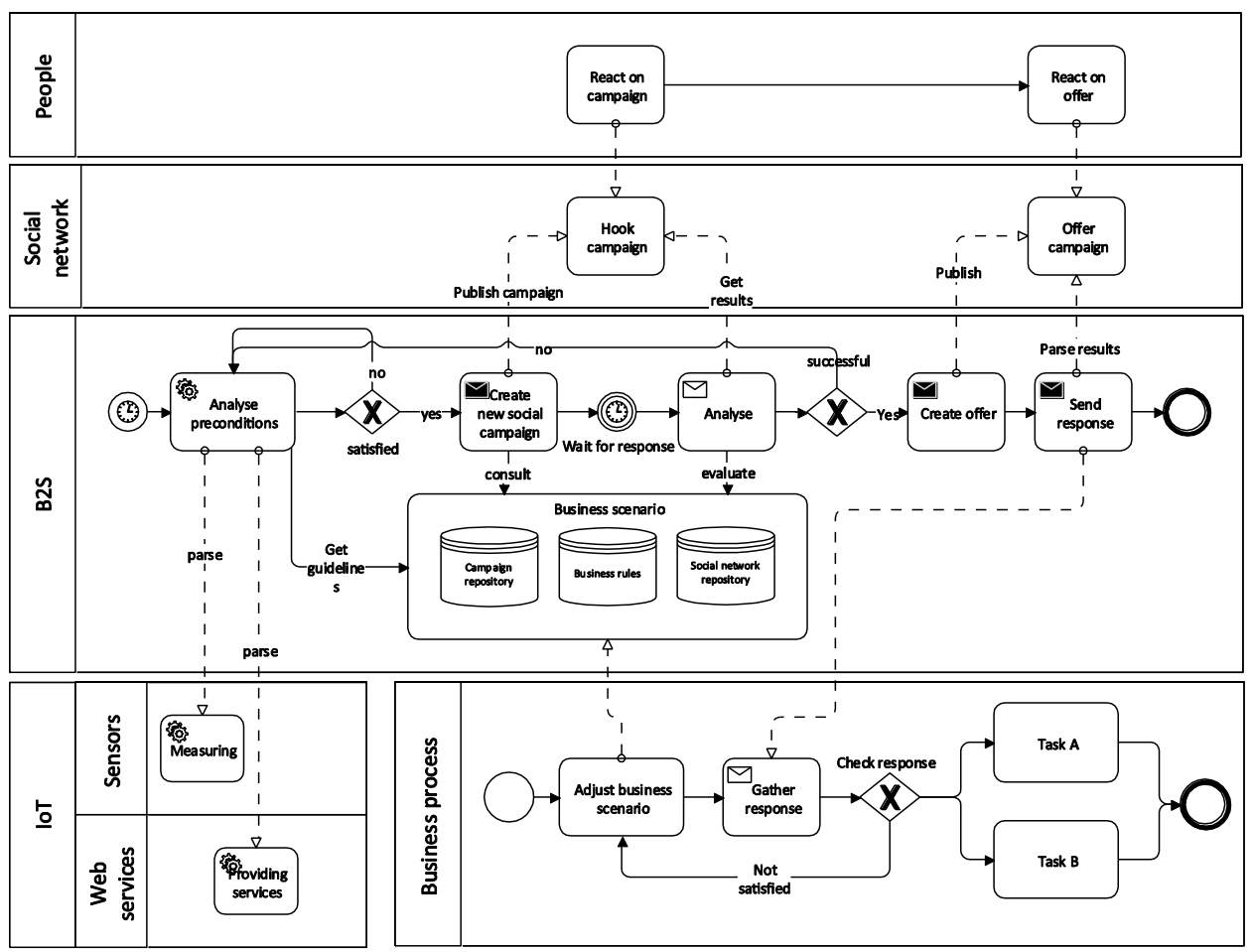

Fig. 3 Dynamics of the business process, B2S and social networks.

\section{IMPLEMENTATION}

To deploy the social world, we use Facebook as a network of choice since it allows anyone to create a Facebook Page ${ }^{18}$ to initiate interactions with its subscribers. Facebook has introduced Graph API ${ }^{19}$ to allow publishing onto Facebook using custom applications. Finally, Facebook offers a service called Webhooks ${ }^{20}$ that notifies us about any feedback on initial SCTO social actions. The details about collected feedback are stored in the SCTO database with their respective date and time, executor details, and social action category. In addition to the general social actions that are common to almost all social

\footnotetext{
${ }^{18}$ www.facebook.com/business/products/pages.

${ }^{19}$ developers.facebook.com/docs/graph-api.

${ }^{20}$ developers.facebook.com/docs/graph-api/webhooks.
} 
networks, Facebook gives the possibility of expressing emotions and/or feelings. These expressions could be Like, HaHa, WoW, Love, Angry, and Sad. Having several Angry reactions to a social action would definitely alert that something went wrong, for example. In order to deploy IoT world, we use the OpenWeatherMaps ${ }^{21}$ web service to retrieve weather forecast data for the city of Novi Pazar.

The Web interface/dashboard of the B2S is available at https://social.connect.rs/b2s/ it is hosted on a Linux Apache server (username/password: manager/manager). The user interface is created in HTML, CSS, JavaScript, and Bootstrap library. The platform's backend is developed in PHP and MySQL. The weather forecast module collects and displays data from Open Weather Map API service. It is developed in the form of php parser that parses JSON formatted data from this service and stores it in a MySQL database once a day using cron job (time-based job scheduler in Unix-like computer operating systems). Novi Pazar weather forecast data for the following 15 days are gathered and stored in the SCTO weather data database. This weather data is later used for comparison with defined preconditions.

The campaigns module allows populating two types of campaigns in the campaigns repository: hook and selling. Once created, a campaign can be reused for an unlimited number of times in different scenarios.

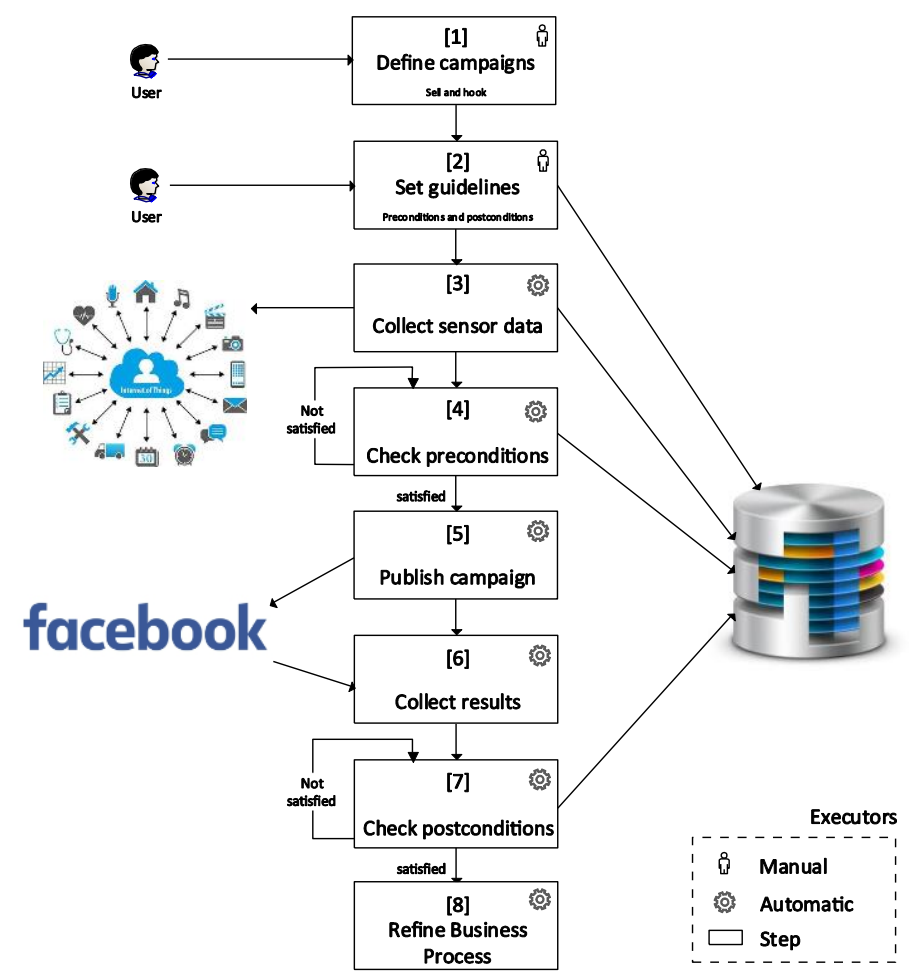

Fig. 4 Chronology of operations in the B2S

\footnotetext{
${ }^{21}$ openweathermap.org/api.
} 
To allow the campaigns to be published on Facebook by B2S, a user needs to create scenarios consisting of 4 parts: precondition, hook campaign, postcondition, and selling campaign (Fig. 6). First of all, precondition and postcondition are stored in the scenarios database. On the other hand, both hook and selling campaigns are stored in the social actions copy database.

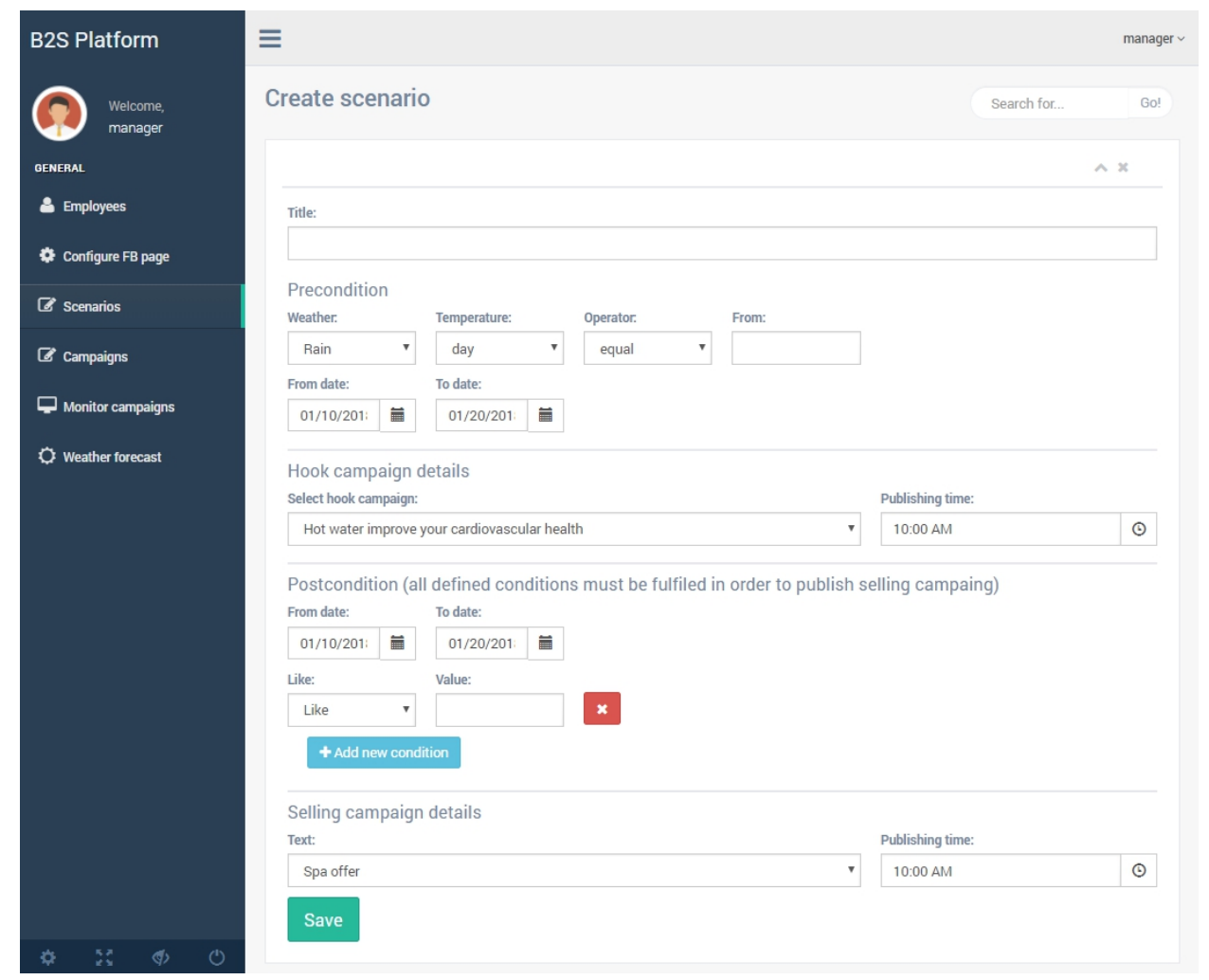

Fig. 6 Creating a scenario

The scenario module allows users to create unlimited number of scenarios in advance, so a user can cover all the events of interest. Once a day, the system is checking scenarios preconditions in order to run the necessary hook campaign. If preconditions are fulfilled, the selected campaign will be published at a scheduled time on the SCTO Facebook page. The feedback on the published hook campaign is tracked and collected using Facebook Webhooks, which is subscribed to the SCTO Facebook page, during the defined timeframe in postconditions. All social actions (Like, Wow, Love...) are collected and stored in SCTO websites' "social responses" database, and are available to the user via a monitoring module at any time (Fig. 7). 

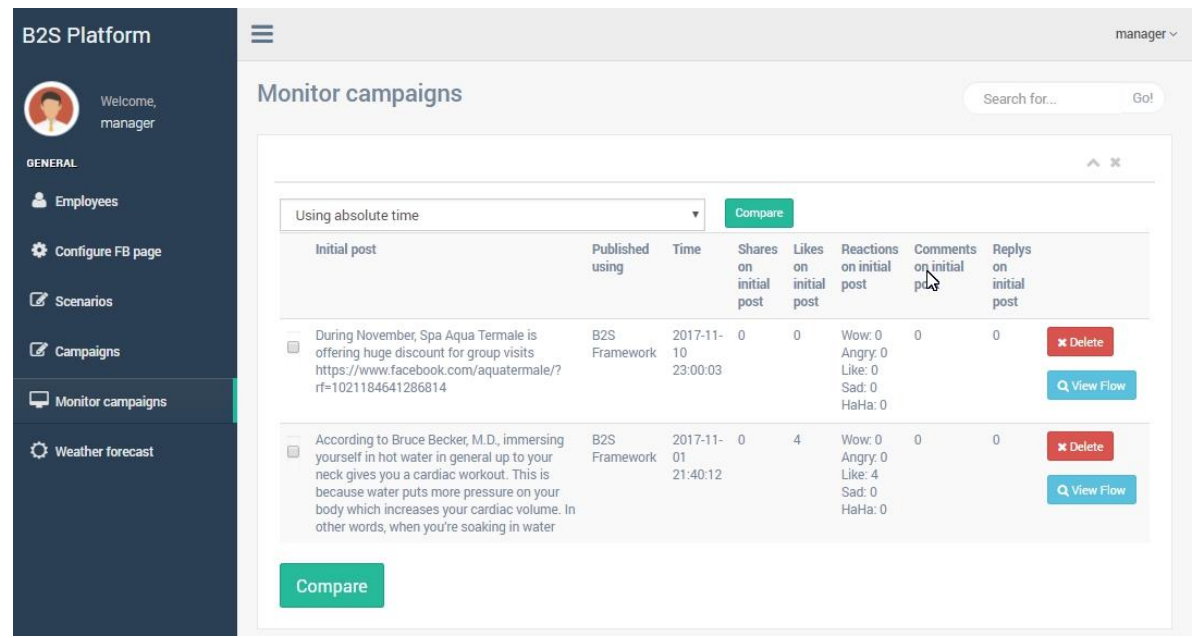

Fig. 7 Monitoring citizens' reactions

Each time the system receives a response from Facebook using Webhooks (subscribers triggered social actions), the system checks if the necessary postconditions are fulfilled to publish the selected "selling" campaign and to send a signal to smart city business process. The business process could be altered using the data received or it could start a new task. The system allows for creating all scenarios in advance with no need for user intervention during the covered period. The system is not limited by the number of created campaigns or scenarios.

\section{CONCLUSION AND FUtURE RESEARCH DiRECTIONS}

In this paper, we presented how business processes in a Smart City could be improved and automated using knowledge gathered from social media, web services as well as from IoT. IoT sensor data and data from web services are used as a trigger for publishing social actions on a social network. Our approach is to initiate social actions and then process and analyze responses from known citizens/visitors, subscribers to our page. In addition to that, we are targeting specific niche in which any of our social action is closely related to our subscribers' interest, and thus, feedback that we collect, has more quality and can be used as a trigger for changing business processes. This way we have control of who we refer to, what our expectation is, how long will we wait to get feedback, and what kind of response is important to us. The feedback collected can be used to start a new or change/adapt existing business process in a smart city. Developing a metric for measuring social actions is part of our future work; currently we are working on the quantification of social actions and the setting of desired expectations. The defined guidelines are leading the process, and satisfying those guidelines can lead to change, adaptation or improvement of a business process or triggering actuators which could affect Smart City component behavior. At this moment we use Facebooks' reactions, for a better analysis of feedback without the need to use data mining. Data mining of social actions content such as comment and reply, as well as triggering actuator are not examined right now and will be in the future. 


\section{REFERENCES}

[1] Albino, V., Berardi, U., \& Dangelico, R. M. (2015). Smart cities: Definitions, dimensions, performance, and initiatives. Journal of Urban Technology, 22(1), 3-21.

[2] Angelidou, M. (2016). Four European smart city strategies. International Journal of Social Science Studies, 4(4), 18-30.

[3] Bauman, K., Tuzhilin, A., \& Zaczynski, R. (2017). Using Social Sensors for Detecting Emergency Events: A Case of Power Outages in the Electrical Utility Industry. ACM Transactions on Management Information Systems (TMIS), 8(2-3), 7.

[4] Facebook.com https://investor.fb.com/investor-news/press-release-details/2016/Facebook-Reports-ThirdQuarter-2016-Results/default.aspx.

[5] Kaplan, Andreas M., and Michael Haenlein. "Users of the world, unite! The challenges and opportunities of Social Media." Business horizons 53.1 (2010): 59-68.

[6] Marsa-Maestre, Ivan, et al. "Mobile agents for service personalization in smart environments." Journal of Networks 3.5 (2008): 30-41.

[7] Mainka, A., Hartmann, S., Stock, W. G., \& Peters, I. (2014, January). Government and social media: A case study of 31 informational world cities. In System Sciences (HICSS), 2014 47th Hawaii International Conference on (pp. 1715-1724). IEEE.

[8] Cosgrave, Ellie, Kate Arbuthnot, and Theo Tryfonas. "Living labs, innovation districts and information marketplaces: A systems approach for smart cities." Procedia Computer Science 16 (2013): 668-677.

[9] Lee, J. H., Hancock, M. G., \& Hu, M. C. (2014). Towards an effective framework for building smart cities: Lessons from Seoul and San Francisco. Technological Forecasting and Social Change, 89, 80-99.

[10] Lee, J. H., Phaal, R., \& Lee, S. H. (2013). An integrated service-device-technology roadmap for smart city development. Technological Forecasting and Social Change, 80(2), 286-306.

[11] Puiu, D., Barnaghi, P., Toenjes, R., Kümper, D., Ali, M. I., Mileo, A., ... \& Gao, F. (2016). Citypulse: Large scale data analytics framework for smart cities. IEEE Access, 4, 1086-1108.

[12] Practical Lessons From the Deployment and Management of a Smart City Internet-of-Things Infrastructure: The SmartSantander Testbed Case http://ieeexplore.ieee.org/abstract/document/7968429/

[13] Chaniotakis, Emmanouil, Constantinos Antoniou, and Francisco Pereira. "Mapping Social Media for Transportation Studies." IEEE Intelligent Systems 31.6 (2016): 64-70.

[14] Cranshaw, J., Schwartz, R., Hong, J. I., \& Sadeh, N. (2012). The livehoods project: Utilizing social media to understand the dynamics of a city.

[15] Badii, A., Carboni, D., Pintus, A., Piras, A., Serra, A., Tiemann, M., \& Viswanathan, N. (2013). CityScripts: Unifying Web, IoT and Smart City Services in a Smart Citizen Workspace. JoWUA, 4(3), 58-78.

[16] Maamar, Z., Burégio, V., Faci, N., Benslimane, D., \& Sheng, Q. Z. (2015, September). " Controlling" Web 2.0 Applications in the Workplace. In Enterprise Distributed Object Computing Conference (EDOC), 2015 IEEE 19th International (pp. 191-200). 